\title{
Chemical stratification in the Orion Bar: JCMT spectral legacy survey observations (Corrigendum)
}

\author{
M. H. D. van der Wiel ${ }^{1,2}$, F. F. S. van der Tak ${ }^{2,1}$, V. Ossenkopf ${ }^{3,2}$, M. Spaans ${ }^{1}$, H. Roberts ${ }^{4}$, \\ G. A. Fuller ${ }^{5}$, and R. Plume ${ }^{6}$
}

\footnotetext{
1 Kapteyn Astronomical Institute, PO Box 800, 9700 AV, Groningen, The Netherlands e-mail: wiel@astro.rug.nl

2 SRON Netherlands Institute for Space Research, PO Box 800, 9700 AV, Groningen, The Netherlands

I. Physikalisches Institut der Universität zu Köln, Zülpicher Straße 77, 50937 Köln, Germany

4 Astrophysics Research Centre, School of Mathematics and Physics, Queen's University of Belfast, Belfast, BT7 1NN, UK

5 Jodrell Bank Centre for Astrophysics, Alan Turing Building, University of Manchester, Manchester, M13 9PL, UK

6 Department of Physics and Astronomy, University of Calgary, Calgary, T2N 1N4, AB, Canada
}

A\&A 498, 161-165 (2009), DOI: 10.1051/0004-6361/200811391

\section{ABSTRACT}

\begin{abstract}
The integrated line intensities in Table 1 of Van der Wiel et al. (2009, A\&A 498, 161) should be modified due to an error in the adopted channel widths: values are reduced by $\sim 20 \%$ for $\mathrm{C}_{2} \mathrm{H}, \mathrm{H}_{2} \mathrm{CO}$ and $\mathrm{SO}$, reduced by a factor $\sim 5$ for $\mathrm{C}^{18} \mathrm{O}$ and $\mathrm{HCN}$, and increased by $\sim 40 \%$ for ${ }^{13} \mathrm{CO}$. Derived column densities for $\mathrm{C}_{2} \mathrm{H}, \mathrm{H}_{2} \mathrm{CO}$ and $\mathrm{SO}$ are decreased by $\sim 20 \%$; for $\mathrm{C}^{18} \mathrm{O}$ it is increased by $\sim 40 \%$. Corrected abundance values of $\mathrm{C}_{2} \mathrm{H}, \mathrm{H}_{2} \mathrm{CO}$ and $\mathrm{SO}$ are a factor $<2$ smaller than the originally published values. The values for $\mathrm{HCN}$ column densities and abundance are replaced by lower limits. Consequences for the discussion and conclusions are minor. In addition, the southwest arrow in Fig. 1 should be shifted to the southwest by $\sim 10^{\prime \prime}$.
\end{abstract}

Key words. ISM: molecules - ISM: structure - ISM: individual: Orion Bar - stars: formation - errata, addenda

\section{Numerical values in Table 1}

Due to an error in the adopted spectral channel widths, the integrated line intensities obtained in the original publication (Van der Wiel et al. 2009) should be corrected by the following factors: 0.84 for $\mathrm{C}_{2} \mathrm{H}, 0.83$ for $\mathrm{H}_{2} \mathrm{CO}, 0.85$ for $\mathrm{SO}, 0.21$ for $\mathrm{C}^{18} \mathrm{O}, 0.18$ for $\mathrm{HCN}$, and 1.41 for ${ }^{13} \mathrm{CO}$.

The values in the last three columns in Table 1 of Van der Wiel et al. (2009) should be updated following Table 1 of this erratum. Note that the HCN column densities are now lower limits, and the limits to the ${ }^{13} \mathrm{CO}$ column density are unchanged. The reduced line strength for $\mathrm{C}^{18} \mathrm{O}$ allows for an optically thin column density estimate. The new $\mathrm{C}^{18} \mathrm{O}$ column density is $\sim 40 \%$ higher than the original, optically thick, estimate.

In the caption of Fig. 1, values for maximum integrated intensity for each map should be: $13.3 \mathrm{~K} \mathrm{~km} \mathrm{~s}^{-1}$ for $\mathrm{C}_{2} \mathrm{H}, 8.7$ for $\mathrm{H}_{2} \mathrm{CO}, 7.7$ for $\mathrm{SO}, 19.9$ for $\mathrm{C}^{18} \mathrm{O}, 70.5$ for $\mathrm{HCN}$, and 276.8 for ${ }^{13} \mathrm{CO}$. The figure is reproduced in this erratum (Fig. 1) with the corrected numbers in the caption.

The vertical scaling in the original Fig. 2 should change by the factors mentioned above for the respective molecules (Fig. 2 in this erratum).

The derived $N\left(\mathrm{H}_{2}\right)$ in Sect. 3, last paragraph, is now $1.0 \times 10^{23} \mathrm{~cm}^{-2}$. The resulting molecular abundances are: $x\left(\mathrm{C}_{2} \mathrm{H}\right)=2 \times 10^{-9}, x\left(\mathrm{H}_{2} \mathrm{CO}\right)=4 \times 10^{-10}, x(\mathrm{SO})=7 \times 10^{-10}$, $x(\mathrm{HCN})>5 \times 10^{-10}$.

The following parts of the discussion (Sect. 4) are affected. The $\mathrm{C}_{2} \mathrm{H}$ abundance discrepancy decreases. Somewhat
Table 1. Corrected values for integrated line strength and derived column densities of observed molecular lines.

\begin{tabular}{|c|c|c|c|c|}
\hline \multirow[t]{2}{*}{ Molecule } & \multirow[t]{2}{*}{ Transition } & \multirow{2}{*}{$\underset{\left(\mathrm{K} \mathrm{km} \mathrm{s}^{-1}\right)}{\int_{\mathrm{mb}} \mathrm{d} v^{a}}$} & \multicolumn{2}{|c|}{$N_{\mathrm{mb}}\left(\mathrm{cm}^{-2}\right)^{b}$} \\
\hline & & & $n=10^{5} \mathrm{~cm}^{-3}$ & LTE \\
\hline $\mathrm{C}_{2} \mathrm{H}$ & $4_{7 / 2} \rightarrow 3_{5 / 2}$ & $8.6 \pm 1.6$ & $4.4 \times 10^{14}$ & $2.3 \times 10^{14}$ \\
\hline $\mathrm{C}_{2} \mathrm{H}$ & $4_{9 / 2} \rightarrow 3_{7 / 2}$ & $11.3 \pm$ & $4.9 \times 10^{14}$ & $2.4 \times 10^{14}$ \\
\hline $\mathrm{o}-\mathrm{H}_{2} \mathrm{CO}$ & $5_{15} \rightarrow 4_{14}$ & $9.3 \pm 1.6$ & $7.7 \times 10^{14}$ & $3.8 \times 10^{13}$ \\
\hline SO & $8_{9} \rightarrow 7_{8}$ & $5.2 \pm 0.9$ & $1.4 \times 10^{15}$ & $7.2 \times 10^{13}$ \\
\hline $\mathrm{C}^{18} \mathrm{O}$ & $\rightarrow 1$ & $20.3 \pm 3.4$ & $2.3 \times 10^{16}$ & $2.9 \times 10^{16}$ \\
\hline $\mathrm{HCN}$ & $\rightarrow 2$ & $74.0 \pm 14$ & $>6.5 \times 10^{12 c}$ & $>4.9 \times 10^{13 c}$ \\
\hline${ }^{13} \mathrm{CO}$ & $\rightarrow 2$ & $186 \pm 32$ & $>2.1 \times 10^{16 c}$ & $>2.5 \times 10^{16 c}$ \\
\hline
\end{tabular}

Notes. ${ }^{(a)}$ Fitted value of integrated intensity at peak position along the northeast slice (see Fig. 1); uncertainties include fitting uncertainty and $15 \%$ absolute calibration uncertainty. ${ }^{(b)}$ Beam-averaged column densities for $n=10^{5} \mathrm{~cm}^{-3}$ and in LTE, both at $T=85 \mathrm{~K}$. ${ }^{(c)}$ Column density uncertain due to high optical depth; values listed here are lower limits.

fortuitously, the discrepancy between modeled and observed $\mathrm{H}_{2} \mathrm{CO}$ abundance almost vanishes if the "observed" $\mathrm{H}_{2} \mathrm{CO}$ column density is derived based on the LTE assumption; in the likely case that $\mathrm{H}_{2} \mathrm{CO}$ is not completely in LTE, the gas-phase model still underestimates its abundance. The SO abundance discrepancy increases to a factor $1.4 \times 10^{4}$. 

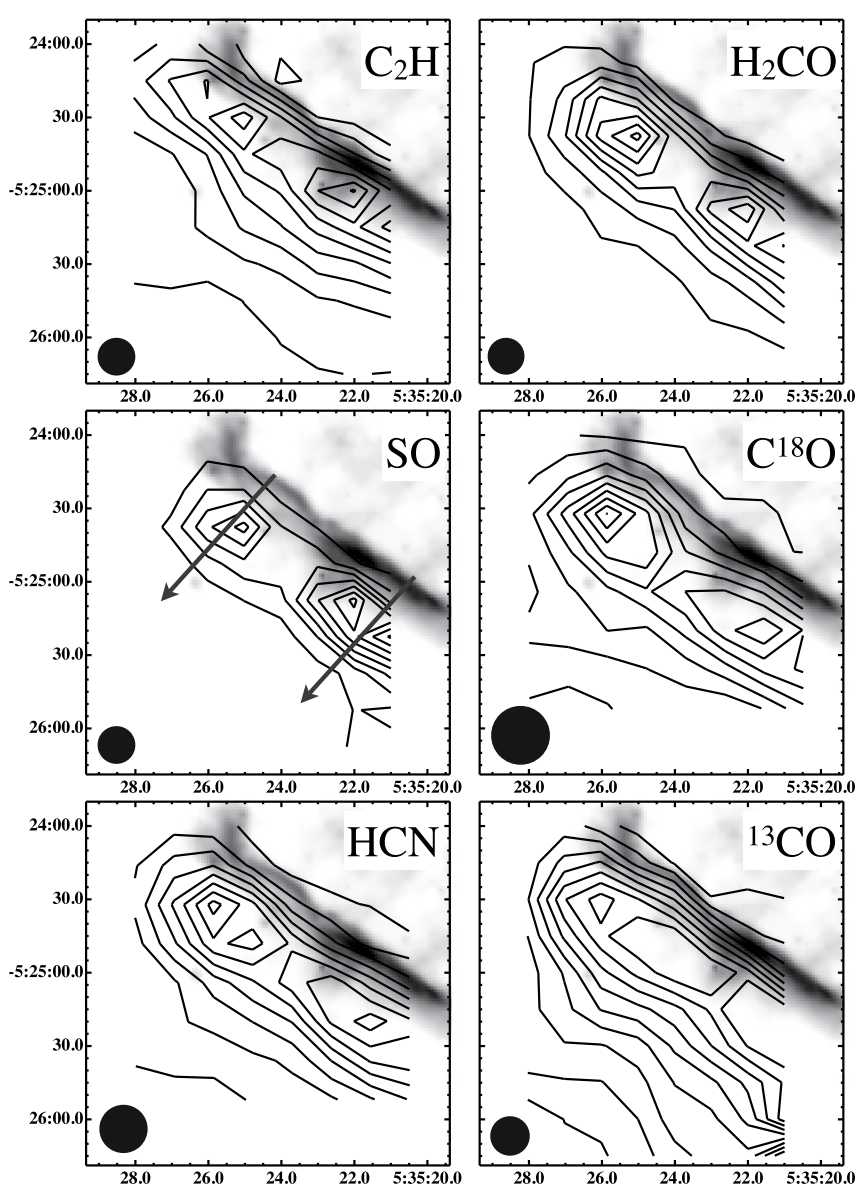

Fig. 1. Images of molecular emission, observed with the JCMT toward the Orion Bar of the species and transitions listed in Table 1. Line intensities are integrated over $5-8 \mathrm{~km} \mathrm{~s}^{-1}$, depending on the line width. The $\mathrm{C}_{2} \mathrm{H}$ transition shown here is the $4_{9 / 2}-3_{7 / 2}$ transition; the other transition shows a similar spatial distribution. Contour levels are drawn at $10,20, \ldots, 90 \%$ of the maximum integrated intensity for every map: $13.3 \mathrm{~K} \mathrm{~km} \mathrm{~s}^{-1}$ for $\mathrm{C}_{2} \mathrm{H}, 8.7$ for $\mathrm{H}_{2} \mathrm{CO}, 7.7$ for $\mathrm{SO}, 19.9$ for $\mathrm{C}^{18} \mathrm{O}$, 70.5 for $\mathrm{HCN}$, and 276.8 for ${ }^{13} \mathrm{CO}$. Note that the measured maximum $\int T_{\mathrm{mb}} \mathrm{d} v$ values across the northeast slices (Table 1) are not the same as the highest value across the entire map. The grayscale background image shows Spitzer Space Telescope $8 \mu \mathrm{m}$ continuum emission. The filled circle in each frame indicates the beam size at the relevant frequency. Axes are annotated with right ascension and declination (J2000).

\section{Arrow in Fig. 1}

The arrow in Fig. 1 indicating the southwest slice is misplaced. It should go through the southwest peak of the $\mathrm{C}^{18} \mathrm{O}$ emission (coordinates RA $=5^{\mathrm{h}} 35^{\mathrm{m}} 21^{\mathrm{s}} \cdot 6, \delta=-5^{\circ} 25^{\prime} 19^{\prime \prime}$, as correctly
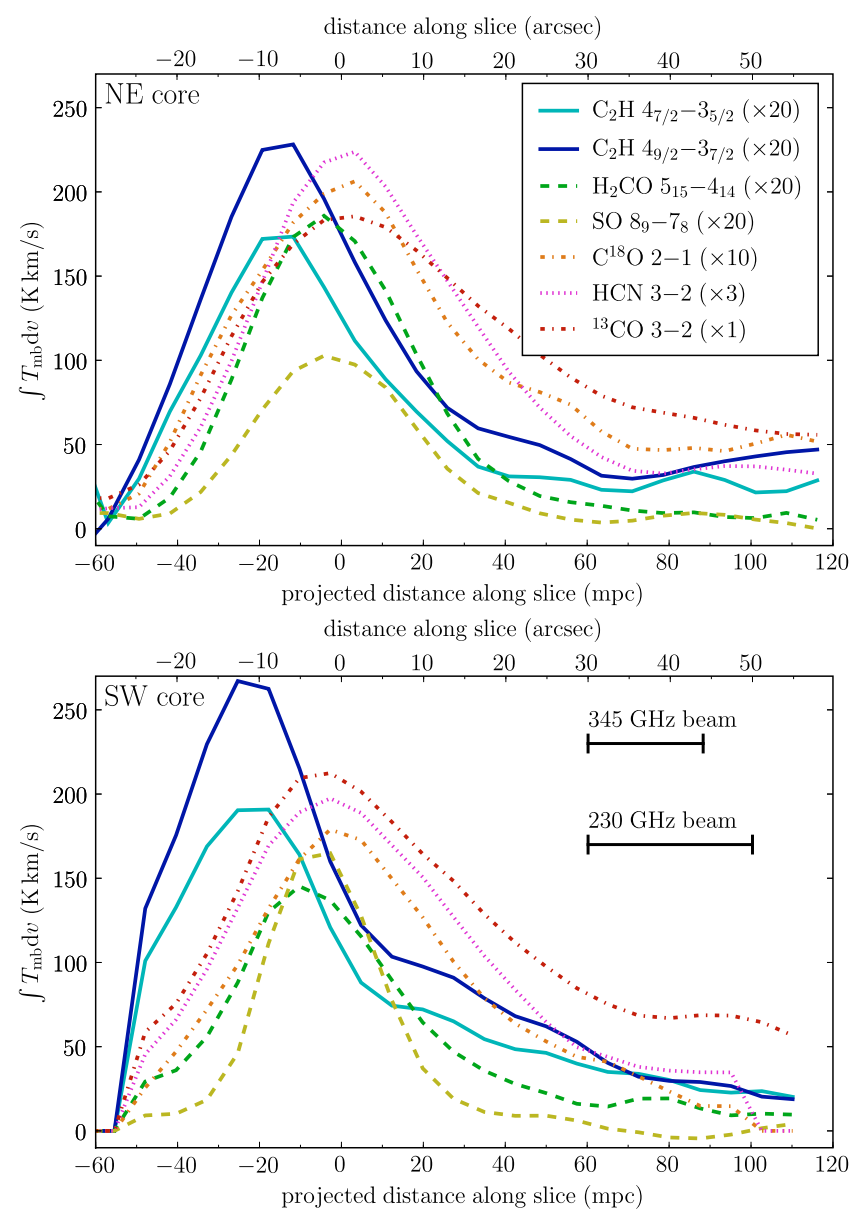

Fig. 2. Profiles of molecular emission along slices perpendicular to the ionization front at position angle $225^{\circ}$ (see arrows in Fig. 1): (top panel) through the northeast core; (bottom panel) through the southwest core. The zero point of the distance scale is placed at the $\mathrm{C}^{18} \mathrm{O}$ peak position: $\left(\mathrm{RA}=5^{\mathrm{h}} 35^{\mathrm{m}} 25^{\mathrm{s}} .4, \delta=-5^{\circ} 24^{\prime} 37^{\prime \prime}\right)$ for the northeast slice and (RA = $\left.5^{\mathrm{h}} 35^{\mathrm{m}} 21^{\mathrm{s}} \cdot 6, \delta=-5^{\circ} 25^{\prime} 19^{\prime \prime}\right)$ for the southwest slice.

stated in the caption of Fig. 2) instead of through the SO emission peak. The correct position of the southwest arrow is shown in Fig. 1 of this erratum.

Acknowledgements. The authors would like to thank Isidore Bedikoglou and Markus Cubick for noting the errors in our original publication.

\section{References}

Van der Wiel, M. H. D., Van der Tak, F. F. S., Ossenkopf, V., et al. 2009, A\&A, 498,161 